\title{
GaBI Journal publishes four issues
} in 2013

\section{Professor Philip Walson, MD}

This issue marks the forth and final issue of the second year's edition of the GaBIJournal. The journal has grown rapidly through the combined efforts of the publisher and her-staff, all of the members of the executive editorial board and the international editorial advisory board, our contributors, advertisers and readers for which I would like to express my sincere appreciation.

Issue 4 begins with two Editorials concerning the possibility that low- to middle-income/ limited resource countries could have a less rigorous biosimilar approval pathway. I wrote the first Editorial in response to the Opinion paper by Ms Barbara Milani and Ms Sara Gaspani concerning the use of pegylated interferon alpha products to treat hepatitis $\mathrm{C}$ that appears later in this issue and the second Editorial by Dr Armando A Genazzani and Dr Nicola Magrini also discusses the more general aspects of this issue and conclude that the solution proposed by Ms Milani and Ms Gaspani may in fact not suit patients in resource-poor countries.

These Editorials are followed by four Letters to the Editor covering a number of important issues including one from Dr Janet S Wyatt who, based on safety and monitoring concerns argues against legislative efforts underway in the US State of California to limit information provided to patients on which biosimilar product they are given. Dr Edward $T$ Maggio then discusses immunological differences as a reason to continue to give unique names to each follow-on biosimilar product. The third letter from Dr Richard O Dolinar who commends the World Health Organization (WHO) for providing distinct International Nonproprietary Names for each biosimilar product. The naming of all follow-on products, especially biosimilars is the subject of considerable clinical, regulatory and proprietary interest and is of importance to everyone concerned with the development, testing, use, approval and monitoring of follow-on products. The final letter from Dr Brendan Shaw corrects some information published as a Perspective by Professor David A Power in Volume 2/Year 2013/Issue 3 of the Generics and Biosimilars Initiative Journal [1]. The paper is about biosimilars licensing and prescribing in Australia.
A Commentary by Dr Gustavo Grampp and Dr Sundar Ramanan on how the quality of biosimilar manufacturing should be maintained including examples of problems that have occurred. They conclude with an interesting proposal for an 'active participation in industry consortia where best practices can be shared among peers'. I have never worked directly for a pharmaceutical company but it seems to me that this suggestion while holding great promise for patients and the healthcare industry if adopted would likely face resistance. I would be interested in hearing what our readers think of this suggestion.

The following Original Research paper by Zhang et al. discusses a related issue, the establishment of reference standards for biosimilar studies.

There are two Review Articles, the first by Vogler et al. explains the components of pharmaceutical expenditures and provides an overview of pharmaceutical policies in Europe, and the second by Dr Vera Brinks discusses the immunogenicity of biosimilar monoclonal antibodies.

In the paper that I wrote an Editorial about Gaspani and Milani in an Opinion suggest that the WHO should develop a system for approving non-biosimilar 'copies' of pegylated interferon alpha for the treatment of hepatitis $\mathrm{C}$ in resource-poor countries. Their suggestion, which is based on their evaluation of the economics of pegylated interferon alpha treatment, suggests that less comparability testing is required in less wealthy than in wealthy countries. We would welcome contributions from our readers on their suggestion as well as my skepticism about the ability of the WHO to do what they suggest as well as my concerns about the outcome of such a second-class approval process.

These papers are followed by a Meeting Report by Professor Mühlebach et al. that summarizes presentations given by an expert panel at an important closed workshop on the clinical and non-clinical aspects of nonbiological complex drugs (NBCDs) held at the 2012 FIP (International Pharmaceutical Federation) Centennial Congress. NBCDs, e.g. liposomes, intravenous iron carbohydrate products and glatiramoids; are a relatively

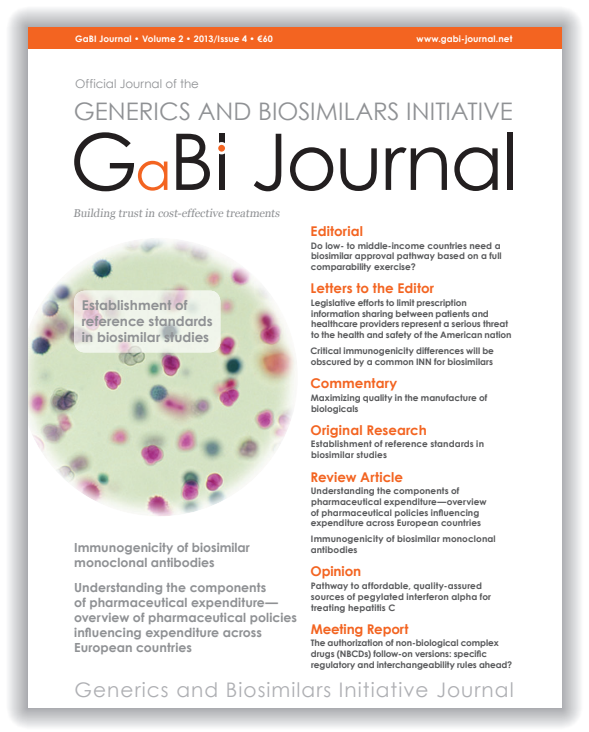

new class of drugs. They are very different and much more complex than are traditional small molecule generics and their composition is highly production method dependent. In fact, while not produced in biological systems they are as or even more complex than many biologicals and can even be impossible to completely characterize by their physiochemical properties. These characteristics raise concerns about the approval of follow-on copies, especially since there is as yet no consistent approach to how they are regulated and approved within or between countries. Members of our editorial board and I feel there is a need for professional education concerning NBCDs. GaBI Journal has therefore begun a number of initiatives aimed at developing best practices for approval, use and monitoring NBCDs. This Meeting Report is the first attempt at bringing this issue to the attention of our readers. Future issues will contain additional information on this subject including meeting reports from educational conferences conducted by GaBI Educational Workshops that will focus on NBCDs.

This issue concludes with a paper on the Japanese regulatory approach to biosimilars.

I want to again urge our readers to submit any comments, commentaries or concerns about the material we publish as well as both their original research and reviews.

Professor Philip Walson, MD Editor-in-Chief, GaBiJournal

\section{Reference}

1. Power D. Licensing and prescribing biosimilars in Australia. Generics and Biosimilars Initiative Journal (GaBI Journal). 2013:2(3):152-4. doi:10.5639/gabij. 2013.0203.030

DOI: $10.5639 /$ gabij.2013.0204.042

Copyright (c) 2013 Pro Pharma Communications International 